\title{
Effect of Organic Amendments, Botanicals and Insecticides against Little Millet Shoot Fly, Atherigona pulla (Wiedemann)
}

\author{
Ravulapenta Sathish", M. Manjunatha and K. Rajashekarappa
}

Department of Agricultural Entomology, University of Agricultural and Horticultural Sciences, Shivamogga, Karnataka, India

*Corresponding author

\section{A B S T R A C T}

\begin{tabular}{|l|}
\hline K e y w o r d s \\
Little millet, Shoot \\
fly management, \\
Organic \\
amendments, \\
Botanicals, \\
Insecticides. \\
\hline Article Info \\
\hline $\begin{array}{l}\text { Accepted: } \\
21 \text { September } 2017 \\
\text { Available Online: } \\
\text { 10 October } 2017\end{array}$ \\
\hline
\end{tabular}

Keywords

Little millet, Shoot fly management, amendments,

\section{Introduction}

Little millet (Panicum sumatrense Roxb.) is an important indigenous crop of Indian Subcontinent. It is grown in Tamil Nadu, Karnataka, Andhra Pradesh, Orissa, Bihar, Madhya Pradesh and Maharashtra. It is locally known as Same or Samai. It matures very early in 70-75 days it serves as an ideal catch crop for multiple and relay cropping systems. It is well known for its drought tolerance and is one of the least water demanding crops. This crop being ecofriendly is highly suitable for sustainable agriculture. The nutritional quality of this grain is superior to fine cereals. Although this crop has best of the attributes, its cultivation sometimes requires attention to manage the only serious pest, shoot fly (Atherigona pulla). This pest alone can cause loss up to 80 per cent or even 100 per cent (Jagadish et al., 1995). Considering seriousness of this pest, an attempt has been made to study the effect of date of sowing on incidence of the shoot fly to predict its occurrence and to develop precise management practices against it.

\section{Materials and Methods}

Experiment was laid out in the Randomized Block Design (RBD) with three replications and nine treatments at Zonal Agricultural and 
Horticultural Research Station (ZAHRS) farm, UAHS, Hiriyur. The little millet variety Sukshema with spacing of $30 \mathrm{~cm}$ between rows was sown during kharif and rabi seasons of 2015-16. All the recommended package of practices was followed except plant protection measures. The treatments one to four viz., neem cake, castor cake, pongamia cake and rice shell ash and treatment eight carbofuran $3 \mathrm{G}$ were applied to soil at the time of sowing. The foliar applications were taken up for the treatments five (NSKE) and six (Azadirachtin) at $7 \mathrm{DAG}$ and at $14 \mathrm{DAG}$. The seeds of treatment seven were treated with imidacloprid $5 \mathrm{~g} / \mathrm{kg}$ seeds, in a plastic bowl uniformly and shade dried. The shade dried treated seeds of all the treatments were sown along with untreated check. The following observations were made during investigation.

\section{Number of eggs per plant}

In each plot, five plants were randomly selected to observe the number of eggs. So, number of eggs was counted from each plant and total number of eggs on five plants (sum of eggs in five randomly selected plants) were taken and averaged to represent the eggs present on each plant.

\section{Deadheart incidence (\%)}

Total number of plants in the each plot and the number of plants showing symptoms of deadhearts were recorded and converted into percentage deadhearts.

Deadheart incidence $(\%)=$ $\frac{\text { Number of plants with deadhearts/plot }}{\text { Total number of plants/plot }}$ X 100

\section{Natural enemies}

The number of coccinellids, chrysopids and spiders present on five randomly selected plants were counted. Total number present on
5 plants were taken and then averaged to get number of coccinellids per plant, number of chrysopids per plant and number of spiders per plant.

\section{Grain yield and fodder yield (q/ha)}

Total grains were collected from each plot by threshing the panicles from each plot. Then the grain and fodder weight of the each plot was recorded by weighing them using electronic balance. Then, it was converted to yield per hectare (quintal).

\section{Cost economics}

The incremental cost benefit ratio (ICBR) was calculated by considering the cost of various treatments, cost incurred for labour, other expenditure (irrigation charges, watch and ward) and market price of little millet grains. Cost effectiveness of each treatment was assessed based on net returns.

Total cost of production included both cultivation as well as plant protection charges.

Gross return $=$ Marketable yield $\mathrm{x}$ Market price

Net return $=$ Grass return - Total cost

Benefit: Cost ratio $=\frac{\text { Net return }}{\text { Total cost }}$

\section{Statistical analysis}

The experimental data recorded on various parameters during the investigation were analyzed statistically by adopting Fischer's method of analysis of variance as outlined by Gomez and Gomez, 1976. The interpretation of data was done by using the critical difference value calculated at 0.05 probability level. The level of significance was expressed at 0.05 probability. 


\section{Results and Discussion}

\section{Number of eggs per plant}

Among the different treatments evaluated against $A$. pulla, seed treatment with imidacloprid @ $5.0 \mathrm{ml} / \mathrm{kg}$ seed (0.49 and 0.27 eggs/plant), soil application of carbofuran $3 \mathrm{G}$ ( 0.83 and 0.55 eggs/plant) were found effective in reducing the oviposition by shoot fly followed by NSKE @ $5 \%$ (1.03 and 0.67 eggs/plant) and azadirachtin @ $1 \%$ (1.26 and0.87 eggs/plant) in both kharif and rabi, respectively (Table 1 ).

Among the organics, all were inferior in reducing the oviposition by shoot fly except neem cake@ 250 kg/ ha (1.31 and 0.98 eggs/plant). The results are in agreement with Tandi and Bajiya (2013) and Parteti et al., (2014) who reported that seed treatment with imidacloprid 600 FS @ $8.75 \mathrm{ml} / \mathrm{kg}$ seed followed by spraying of NSKE 5\% was most effective in reducing the oviposition by shoot fly.

\section{Per cent deadheart}

Same trend as above was followed in case of per cent deadheart. During kharif) and rabi, imidacloprid@5.0 ml/ kg seed (2.41 and $1.52 \%$, respectively) and carbofuran 3G (4.70 and $4.07 \%$, respectively) were significantly superior over organic amendments in reducing the shoot fly incidence (Table 2). None of the organic amendments were effective in reducing shoot fly incidence except rice hull ash (14.11 and 9.70\%, respectively).

The present findings are in conformity with the studies of Kumar and Channaveerswami (2015) and Rajesh et al., (2016) who reported that, seed treatment with imidacloprid 600 FS @ $5 \mathrm{ml} / \mathrm{kg}$ of seed gave better results against shoot fly, A. soccata.

\section{Natural enemy population}

Only predatory coccinellid population was found during the study period. Organic amendments and botanicals conserved significantly highest number of coccinellid population and were followed by untreated check (1.47 coccinellids/ plant) during kharif and rabi.

The insecticides, imidacloprid and carbofuran $3 \mathrm{G}$ conserved very low coccinellid population and inferior among the treatments with 0.18 and 0.29 coccinellids/ plant, respectively during kharif and 0.31 and 0.18 coccinellids/ plant, respectively during rabi (Table 3 ).

These findings are in agreement with Mashwani et al., (2011) and Parteti et al., (2014) who reported imidacloprid seed treatment and carbofuran $3 \mathrm{G}$ to reduce the predatory coccinellid population.

\section{Yield and cost economics}

Highest grain and fodder yield was obtained in imidacloprid 70 WS seed treatment @ 5.0 $\mathrm{ml} / \mathrm{kg}$ seed in both kharif (12.01 and 78.99 q/ha, respectively) and rabi (12.07 and 81.54 q/ha, respectively) and carbofuran 3G @ 10 $\mathrm{kg} / \mathrm{ha}$ with 10.95 and $72.16 \mathrm{q} / \mathrm{h}$ grain and fodder yield, respectively during kharif and 11.56 and $82.89 \mathrm{q} / \mathrm{ha}$ grain and fodder yield, respectively during rabi (Table 4). These were followed by NSKE @ $5 \%$ by recording 10.12 and $62.78 \mathrm{q} / \mathrm{ha}$ grain and fodder yield, respectively during kharif and 10.46 and $72.65 \mathrm{q} / \mathrm{ha}$ grain and fodder yield, respectively during rabi. There was no statistical difference in grain yields among the organics evaluated and ineffective against shoot fly except neem cake. However, untreated check recorded significantly lowest grain and fodder yield of 5.25 and $40.63 \mathrm{q} / \mathrm{ha}$, respectively during kharif whereas 5.96 and $57.55 \mathrm{q} / \mathrm{ha}$, respectively during rabi. 
Table.1 Effect of organic amendments on the oviposition by shoot fly, A. pulla during kharif and rabi (2015-16)

\begin{tabular}{|c|c|c|c|c|c|c|c|c|c|}
\hline \multirow{3}{*}{$\begin{array}{l}\text { Tr. } \\
\text { No. }\end{array}$} & \multirow{3}{*}{ Treatments } & \multicolumn{8}{|c|}{ Number of eggs/ plant } \\
\hline & & \multicolumn{4}{|c|}{ Kharif } & \multicolumn{4}{|c|}{ Rabi } \\
\hline & & 14 DAE & 21 DAE & 28 DAE & Mean & 14 DAE & 21 DAE & 28 DAE & Mean \\
\hline $\mathbf{T}_{1}$ & $\begin{array}{l}\text { Neem cake @ } 250 \text { kg/ ha } \\
(\mathrm{SA})\end{array}$ & $\begin{array}{c}1.47 \\
(1.40)^{\mathrm{bcd}} \\
\end{array}$ & $\begin{array}{c}1.33 \\
(1.35)^{\mathrm{b}}\end{array}$ & $\begin{array}{c}1.13 \\
(1.28)^{\mathrm{cd}}\end{array}$ & $1.31^{\mathrm{b}}$ & $\begin{array}{c}0.87 \\
(1.17)^{\mathrm{b}}\end{array}$ & $\begin{array}{c}1.13 \\
(1.28)^{\mathrm{bcd}} \\
\end{array}$ & $\begin{array}{c}0.93 \\
(1.20)^{\mathrm{bc}}\end{array}$ & $0.98^{\mathrm{cd}}$ \\
\hline $\mathbf{T}_{2}$ & $\begin{array}{l}\text { Castor cake @ } 250 \mathrm{~kg} / \mathrm{ha} \\
(\mathrm{SA})\end{array}$ & $\begin{array}{c}1.77 \\
(1.50)^{\mathrm{abc}}\end{array}$ & $\begin{array}{c}2.00 \\
(1.58)^{\mathrm{a}} \\
\end{array}$ & $\begin{array}{c}1.47 \\
(1.40)^{\mathrm{ab}}\end{array}$ & $1.74^{\mathrm{a}}$ & $\begin{array}{c}1.00 \\
(1.22)^{\mathrm{b}}\end{array}$ & $\begin{array}{c}1.40 \\
(1.38)^{\mathrm{ab}}\end{array}$ & $\begin{array}{c}1.13 \\
(1.28)^{\mathrm{ab}}\end{array}$ & $1.18^{\mathrm{bc}}$ \\
\hline $\mathbf{T}_{\mathbf{3}}$ & $\begin{array}{l}\text { Pongamia cake@ } 250 \text { kg/ ha } \\
(\mathrm{SA})\end{array}$ & $\begin{array}{c}1.83 \\
(1.53)^{\mathrm{ab}} \\
\end{array}$ & $\begin{array}{c}1.93 \\
(1.56)^{\mathrm{a}} \\
\end{array}$ & $\begin{array}{c}1.27 \\
(1.33)^{\mathrm{bc}}\end{array}$ & $1.68^{\mathrm{a}}$ & $\begin{array}{c}0.93 \\
(1.20)^{\mathrm{b}}\end{array}$ & $\begin{array}{c}1.20 \\
(1.30)^{\mathrm{bc}}\end{array}$ & $\begin{array}{c}1.00 \\
(1.22)^{\mathrm{bc}}\end{array}$ & $1.04^{\mathrm{bcd}}$ \\
\hline $\mathbf{T}_{4}$ & Rice hull ash @1t/ ha (SA) & $\begin{array}{c}1.97 \\
(1.57)^{\mathrm{ab}}\end{array}$ & $\begin{array}{c}1.80 \\
(1.52)^{\mathrm{a}}\end{array}$ & $\begin{array}{c}1.47 \\
(1.40)^{\mathrm{ab}}\end{array}$ & $1.74^{\mathrm{a}}$ & $\begin{array}{c}1.00 \\
(1.22)^{\mathrm{b}}\end{array}$ & $\begin{array}{c}1.47 \\
(1.40)^{\mathrm{ab}}\end{array}$ & $\begin{array}{c}1.13 \\
(1.28)^{\mathrm{ab}}\end{array}$ & $1.20^{\mathrm{b}}$ \\
\hline $\mathbf{T}_{5}$ & NSKE@ @ & $\begin{array}{c}1.10 \\
(1.26)^{\mathrm{d}}\end{array}$ & $\begin{array}{c}0.93 \\
(1.20)^{\mathrm{cd}}\end{array}$ & $\begin{array}{c}1.07 \\
(1.25)^{\mathrm{cd}}\end{array}$ & $1.03^{b c}$ & $\begin{array}{c}0.40 \\
(0.94)^{\mathrm{d}}\end{array}$ & $\begin{array}{c}0.87 \\
(1.17)^{\mathrm{d}}\end{array}$ & $\begin{array}{c}0.73 \\
(1.11)^{\mathrm{cd}}\end{array}$ & $0.67^{\mathrm{ef}}$ \\
\hline $\mathbf{T}_{6}$ & Azadirachtin@1\% & $\begin{array}{c}1.30 \\
(1.34)^{\mathrm{cd}} \\
\end{array}$ & $\begin{array}{c}1.33 \\
(1.35)^{\mathrm{b}}\end{array}$ & $\begin{array}{c}1.13 \\
(1.28)^{\mathrm{cd}} \\
\end{array}$ & $1.26^{\mathrm{b}}$ & $\begin{array}{c}0.73 \\
(1.11)^{\mathrm{bc}}\end{array}$ & $\begin{array}{c}1.00 \\
(1.22)^{\mathrm{cd}}\end{array}$ & $\begin{array}{c}0.87 \\
(1.17)^{\mathrm{bc}}\end{array}$ & $0.87^{\mathrm{de}}$ \\
\hline $\mathbf{T}_{7}$ & $\begin{array}{l}\text { Imidacloprid 70 WS @ } 5 \mathrm{~g} / \\
\mathrm{kg} \text { of seed (ST) }\end{array}$ & $\begin{array}{c}0.20 \\
(0.83)^{\mathrm{f}}\end{array}$ & $\begin{array}{c}0.67 \\
(1.08)^{\mathrm{d}}\end{array}$ & $\begin{array}{c}0.60 \\
(1.05)^{\mathrm{e}} \\
\end{array}$ & $0.49^{\mathrm{d}}$ & $\begin{array}{c}0.13 \\
(0.79)^{\mathrm{e}} \\
\end{array}$ & $\begin{array}{c}0.27 \\
(0.87)^{\mathrm{f}}\end{array}$ & $\begin{array}{c}0.40 \\
(0.94)^{\mathrm{e}} \\
\end{array}$ & $0.27^{\mathrm{g}}$ \\
\hline $\mathbf{T}_{8}$ & $\begin{array}{l}\text { Carbofuran 3 G @ } 10 \text { kg/ha } \\
(\mathrm{SA})\end{array}$ & $\begin{array}{c}0.57 \\
(1.03)^{\mathrm{e}}\end{array}$ & $\begin{array}{c}1.07 \\
(1.25)^{\mathrm{bc}}\end{array}$ & $\begin{array}{c}0.87 \\
(1.17)^{\mathrm{d}}\end{array}$ & $0.83^{\mathrm{c}}$ & $\begin{array}{c}0.53 \\
(1.01)^{\mathrm{cd}}\end{array}$ & $\begin{array}{c}0.60 \\
(1.05)^{\mathrm{e}}\end{array}$ & $\begin{array}{c}0.53 \\
(1.02)^{\mathrm{de}}\end{array}$ & $0.55^{\mathrm{f}}$ \\
\hline $\mathbf{T}_{9}$ & Untreated check & $\begin{array}{c}2.07 \\
(1.59)^{\mathrm{a}}\end{array}$ & $\begin{array}{c}2.17 \\
(1.63)^{\mathrm{a}}\end{array}$ & $\begin{array}{c}1.80 \\
(1.52)^{\mathrm{a}}\end{array}$ & $2.01^{\mathrm{a}}$ & $\begin{array}{c}1.73 \\
(1.49)^{\mathrm{a}}\end{array}$ & $\begin{array}{c}1.67 \\
(1.47)^{\mathrm{a}}\end{array}$ & $\begin{array}{c}1.40 \\
(1.38)^{\mathrm{a}}\end{array}$ & $1.60^{\mathrm{a}}$ \\
\hline & & 0.06 & 0.05 & 0.04 & & 0.04 & 0.04 & 0.04 & \\
\hline & & 0.18 & 0.14 & 0.12 & & 0.13 & 0.12 & 0.12 & \\
\hline & & 7.83 & 5.91 & 5.20 & & 6.49 & 5.56 & 5.79 & \\
\hline & & * & $*$ & $*$ & & $*$ & $*$ & $*$ & \\
\hline
\end{tabular}

Figures in the parentheses are $\sqrt{x}+0.5$ transformed values; DAE $=$ Days after emergence.

Means in the columns followed by the same alphabet do not differ significantly by DMRT ( $\mathrm{P}=0.05)$; $\mathrm{SA}=$ Soil application; $\mathrm{ST}=\mathrm{Seed}$ treatment 
Table.2 Effect of organic amendments on the deadheart per cent by shoot fly, A. pulla during kharif and rabi (2015-16)

\begin{tabular}{|c|c|c|c|c|c|c|c|c|c|}
\hline \multirow{3}{*}{$\begin{array}{l}\text { Tr. } \\
\text { No. }\end{array}$} & \multirow{3}{*}{ Treatments } & \multicolumn{8}{|c|}{ Deadheart per cent } \\
\hline & & \multicolumn{4}{|c|}{ Kharif } & \multicolumn{4}{|c|}{ Rabi } \\
\hline & & 14 DAE & 21 DAE & 28 DAE & Mean & 14 DAE & 21 DAE & 28 DAE & Mean \\
\hline $\mathbf{T}_{1}$ & $\begin{array}{l}\text { Neem cake@ } 250 \text { kg/ha } \\
(\mathrm{SA})\end{array}$ & $\begin{array}{c}12.00 \\
(20.19)^{\mathrm{c}}\end{array}$ & $\begin{array}{c}19.11 \\
(25.89)^{\mathrm{c}} \\
\end{array}$ & $\begin{array}{c}27.22 \\
(31.42)^{\mathrm{c}} \\
\end{array}$ & $19.45^{\mathrm{bc}}$ & $\begin{array}{c}8.00 \\
(16.44)^{b}\end{array}$ & $\begin{array}{c}15.78 \\
(23.40)^{b}\end{array}$ & $\begin{array}{c}20.44 \\
(26.89)^{\mathrm{b}} \\
\end{array}$ & $14.74^{\mathrm{b}}$ \\
\hline $\mathbf{T}_{2}$ & $\begin{array}{l}\text { Castor cake @ } 250 \text { kg/ha } \\
(\mathrm{SA})\end{array}$ & $\begin{array}{c}15.44 \\
(23.14)^{b}\end{array}$ & $\begin{array}{c}25.67 \\
(30.45)^{\mathrm{b}}\end{array}$ & $\begin{array}{c}34.56 \\
(36.02)^{\mathrm{ab}}\end{array}$ & $25.22^{b}$ & $\begin{array}{c}11.11 \\
(19.47)^{b}\end{array}$ & $\begin{array}{c}19.67 \\
(26.34)^{b}\end{array}$ & $\begin{array}{c}25.11 \\
(30.09)^{\mathrm{b}}\end{array}$ & $18.63^{b}$ \\
\hline $\mathbf{T}_{3}$ & $\begin{array}{l}\text { Pongamia cake @ } 250 \mathrm{~kg} / \mathrm{ha} \\
(\mathrm{SA})\end{array}$ & $\begin{array}{c}14.89 \\
(22.70)^{\mathrm{b}}\end{array}$ & $\begin{array}{c}23.44 \\
(28.97)^{b}\end{array}$ & $\begin{array}{c}32.00 \\
(34.45)^{\mathrm{bc}}\end{array}$ & $23.44^{\mathrm{b}}$ & $\begin{array}{c}10.22 \\
(18.65)^{b}\end{array}$ & $\begin{array}{c}18.67 \\
(25.61)^{b}\end{array}$ & $\begin{array}{c}24.89 \\
(29.94)^{\mathrm{b}}\end{array}$ & $17.93^{b}$ \\
\hline $\mathbf{T}_{4}$ & Rice hull ash @1t/ ha (SA) & $\begin{array}{c}6.22 \\
(14.43)^{\mathrm{d}}\end{array}$ & $\begin{array}{c}15.44 \\
(23.14)^{\mathrm{d}}\end{array}$ & $\begin{array}{c}20.67 \\
(27.05)^{\mathrm{d}}\end{array}$ & $14.11^{\mathrm{cd}}$ & $\begin{array}{c}4.67 \\
(12.47)^{\mathrm{c}}\end{array}$ & $\begin{array}{c}10.22 \\
(18.60)^{\mathrm{c}}\end{array}$ & $\begin{array}{c}14.22 \\
(22.10)^{\mathrm{c}}\end{array}$ & $9.70^{\mathrm{c}}$ \\
\hline $\mathbf{T}_{5}$ & NSKE@ @ & $\begin{array}{c}4.56 \\
(12.32)^{\mathrm{d}}\end{array}$ & $\begin{array}{c}12.11 \\
(20.31)^{\mathrm{e}}\end{array}$ & $\begin{array}{c}10.78 \\
(19.05)^{f}\end{array}$ & $9.15^{\mathrm{de}}$ & $\begin{array}{c}2.00 \\
(8.06)^{\mathrm{d}}\end{array}$ & $\begin{array}{c}8.55 \\
(16.85)^{\mathrm{c}}\end{array}$ & $\begin{array}{c}12.78 \\
(20.77)^{\mathrm{c}}\end{array}$ & $7.78^{\mathrm{cd}}$ \\
\hline $\mathbf{T}_{6}$ & Azadirachtin@1\% & $\begin{array}{c}4.78 \\
(12.63)^{d}\end{array}$ & $\begin{array}{c}13.56 \\
(21.58)^{\mathrm{de}}\end{array}$ & $\begin{array}{c}15.78 \\
(23.38)^{\mathrm{e}}\end{array}$ & $11.37^{\mathrm{d}}$ & $\begin{array}{c}2.78 \\
(9.59)^{\mathrm{cd}}\end{array}$ & $\begin{array}{c}9.55 \\
(17.88)^{\mathrm{c}}\end{array}$ & $\begin{array}{c}13.33 \\
(21.23)^{\mathrm{c}}\end{array}$ & $8.55^{\mathrm{c}}$ \\
\hline $\mathbf{T}_{7}$ & $\begin{array}{l}\text { Imidacloprid } 70 \text { WS @ } 5 \mathrm{~g} / \\
\mathrm{kg} \text { of seed }(\mathrm{ST})\end{array}$ & $\begin{array}{c}1.33 \\
(6.15)^{\mathrm{f}}\end{array}$ & $\begin{array}{c}2.55 \\
(9.02)^{\mathrm{g}} \\
\end{array}$ & $\begin{array}{c}3.33 \\
(10.47)^{\mathrm{h}} \\
\end{array}$ & $2.41^{\mathrm{f}}$ & $\begin{array}{c}0.33 \\
(1.91)^{\mathrm{e}} \\
\end{array}$ & $\begin{array}{c}1.78 \\
(7.61)^{\mathrm{e}} \\
\end{array}$ & $\begin{array}{c}2.44 \\
(8.98)^{\mathrm{e}} \\
\end{array}$ & $1.52^{\mathrm{e}}$ \\
\hline $\mathbf{T}_{8}$ & $\begin{array}{l}\text { Carbofuran 3G@10 kg/ha } \\
\text { (SA) }\end{array}$ & $\begin{array}{c}2.89 \\
(9.75)^{\mathrm{e}}\end{array}$ & $\begin{array}{c}4.22 \\
(11.83)^{\mathrm{f}}\end{array}$ & $\begin{array}{c}7.00 \\
(15.34)^{\mathrm{g}}\end{array}$ & $4.70^{\mathrm{ef}}$ & $\begin{array}{c}1.89 \\
(6.46)^{\mathrm{d}}\end{array}$ & $\begin{array}{c}4.22 \\
(11.86)^{\mathrm{d}}\end{array}$ & $\begin{array}{c}6.11 \\
(14.26)^{\mathrm{d}}\end{array}$ & $4.07^{\mathrm{de}}$ \\
\hline \multirow[t]{5}{*}{$\mathbf{T}_{9}$} & Untreated check & $\begin{array}{c}21.89 \\
(27.88)^{\mathrm{a}}\end{array}$ & $\begin{array}{c}34.56 \\
(36.02)^{\mathrm{a}}\end{array}$ & $\begin{array}{c}38.78 \\
(38.53)^{\mathrm{a}}\end{array}$ & $31.74^{\mathrm{a}}$ & $\begin{array}{c}16.89 \\
(24.26)^{\mathrm{a}}\end{array}$ & $\begin{array}{c}24.33 \\
(29.57)^{\mathrm{a}}\end{array}$ & $\begin{array}{c}31.67 \\
(34.26)^{\mathrm{a}}\end{array}$ & $24.30^{\mathrm{a}}$ \\
\hline & & 0.81 & 0.82 & 1.08 & & 1.31 & 0.99 & 1.17 & \\
\hline & & 2.44 & 2.46 & 3.23 & & 3.91 & 2.97 & 3.51 & \\
\hline & & 8.50 & 6.17 & 7.13 & & 17.35 & 8.68 & 8.76 & \\
\hline & & $*$ & * & $*$ & & $*$ & $*$ & $*$ & \\
\hline
\end{tabular}

Figures in the parentheses are $\sqrt{x}+0.5$ transformed values; $D A E=$ Days after emergence.

Means in the columns followed by the same alphabet do not differ significantly by DMRT $(\mathrm{P}=0.05)$; SA=Soil application; $\mathrm{ST}=$ Seed treatment 
Table.3 Effect of organic amendments on coccinellid population in little millet ecosystem during kharif and rabi (2015-16)

\begin{tabular}{|c|c|c|c|c|c|c|c|c|c|}
\hline \multirow{3}{*}{$\begin{array}{l}\text { Tr. } \\
\text { No. }\end{array}$} & \multirow{3}{*}{ Treatments } & \multicolumn{8}{|c|}{ Number of coccinellids/ plant } \\
\hline & & \multicolumn{4}{|c|}{ Kharif } & \multicolumn{4}{|c|}{ Rabi } \\
\hline & & 14 DAE & 21 DAE & 28 DAE & Mean & 14 DAE & 21 DAE & 28 DAE & Mean \\
\hline $\mathbf{T}_{1}$ & $\begin{array}{l}\text { Neem cake@ } 250 \mathrm{~kg} / \mathrm{ha} \\
(\mathrm{SA})\end{array}$ & $\begin{array}{c}1.07 \\
(1.25)^{\mathrm{abc}}\end{array}$ & $\begin{array}{c}1.27 \\
(1.33)^{b}\end{array}$ & $\begin{array}{c}1.47 \\
(1.40)^{\mathrm{a}}\end{array}$ & $1.27^{\mathrm{abc}}$ & $\begin{array}{c}1.40 \\
(1.37)^{\mathrm{abc}}\end{array}$ & $\begin{array}{c}1.27 \\
(1.33)^{\mathrm{b}}\end{array}$ & $\begin{array}{c}1.57 \\
(1.44)^{\mathrm{a}}\end{array}$ & $1.41^{\mathrm{b}}$ \\
\hline $\mathbf{T}_{2}$ & $\begin{array}{l}\text { Castor cake @ } 250 \text { kg/ha } \\
(\mathrm{SA})\end{array}$ & $\begin{array}{c}0.87 \\
(1.17)^{\mathrm{cd}}\end{array}$ & $\begin{array}{c}0.73 \\
(1.11)^{\mathrm{d}} \\
\end{array}$ & $\begin{array}{c}0.80 \\
(1.14)^{\mathrm{d}} \\
\end{array}$ & $0.80^{\mathrm{e}}$ & $\begin{array}{c}1.00 \\
(1.22)^{\mathrm{bcd}}\end{array}$ & $\begin{array}{c}1.20 \\
(1.30)^{\mathrm{b}}\end{array}$ & $\begin{array}{c}1.20 \\
(1.30)^{\mathrm{bc}}\end{array}$ & $1.13^{\mathrm{b}}$ \\
\hline $\mathbf{T}_{\mathbf{3}}$ & $\begin{array}{l}\text { Pongamia cake @ } 250 \mathrm{~kg} / \mathrm{ha} \\
(\mathrm{SA})\end{array}$ & $\begin{array}{c}0.93 \\
(1.20)^{\mathrm{bc}}\end{array}$ & $\begin{array}{c}1.00 \\
(1.22)^{\mathrm{c}}\end{array}$ & $\begin{array}{c}1.07 \\
(1.25)^{\mathrm{cd}}\end{array}$ & $1.00^{\text {cde }}$ & $\begin{array}{c}1.30 \\
(1.34)^{\mathrm{abcd}}\end{array}$ & $\begin{array}{c}1.20 \\
(1.30)^{\mathrm{b}}\end{array}$ & $\begin{array}{c}1.20 \\
(1.30)^{\mathrm{bc}}\end{array}$ & $1.23^{\mathrm{b}}$ \\
\hline $\mathbf{T}_{4}$ & Rice hull ash @1t/ha (SA) & $\begin{array}{c}1.20 \\
(1.30)^{\mathrm{ab}}\end{array}$ & $\begin{array}{c}1.40 \\
(1.38)^{\mathrm{ab}}\end{array}$ & $\begin{array}{c}1.33 \\
(1.35)^{\mathrm{abc}}\end{array}$ & $1.31^{\mathrm{ab}}$ & $\begin{array}{c}1.47 \\
(1.40)^{\mathrm{ab}}\end{array}$ & $\begin{array}{c}1.27 \\
(1.33)^{\mathrm{b}}\end{array}$ & $\begin{array}{c}1.13 \\
(1.28)^{\mathrm{c}}\end{array}$ & $1.29^{\mathrm{b}}$ \\
\hline $\mathbf{T}_{5}$ & NSKE@ @ & $\begin{array}{c}0.53 \\
(1.02)^{\mathrm{e}}\end{array}$ & $\begin{array}{c}1.33 \\
(1.35)^{\mathrm{ab}}\end{array}$ & $\begin{array}{c}1.40 \\
(1.38)^{\mathrm{ab}}\end{array}$ & $1.09^{\mathrm{bcd}}$ & $\begin{array}{c}0.87 \\
(1.17)^{\mathrm{d}}\end{array}$ & $\begin{array}{c}1.40 \\
(1.38)^{\mathrm{b}}\end{array}$ & $\begin{array}{c}1.43 \\
(1.39)^{\mathrm{ab}}\end{array}$ & $1.23^{\mathrm{b}}$ \\
\hline $\mathbf{T}_{6}$ & Azadirachtin@1\% & $\begin{array}{c}0.67 \\
(1.08)^{\text {de }} \\
\end{array}$ & $\begin{array}{c}1.13 \\
(1.28)^{\mathrm{bc}}\end{array}$ & $\begin{array}{c}1.07 \\
(1.25)^{\mathrm{bcd}} \\
\end{array}$ & $0.96^{\mathrm{de}}$ & $\begin{array}{c}0.93 \\
(1.20)^{\mathrm{cd}}\end{array}$ & $\begin{array}{c}1.27 \\
(1.33)^{\mathrm{b}}\end{array}$ & $\begin{array}{c}1.40 \\
(1.38)^{\mathrm{abc}}\end{array}$ & $1.20^{\mathrm{b}}$ \\
\hline $\mathbf{T}_{7}$ & $\begin{array}{l}\text { Imidacloprid 70 WS @ } 5 \mathrm{~g} / \\
\mathrm{kg} \text { of seed (ST) }\end{array}$ & $\begin{array}{c}0.07 \\
(0.75)^{\mathrm{f}}\end{array}$ & $\begin{array}{c}0.20 \\
(0.83)^{\mathrm{e}}\end{array}$ & $\begin{array}{c}0.27 \\
(0.87)^{\mathrm{e}} \\
\end{array}$ & $0.18^{\mathrm{f}}$ & $\begin{array}{c}0.27 \\
(0.87)^{\mathrm{e}} \\
\end{array}$ & $\begin{array}{c}0.33 \\
(0.91)^{\mathrm{c}}\end{array}$ & $\begin{array}{c}0.33 \\
(0.91)^{\mathrm{d}} \\
\end{array}$ & $0.31^{\mathrm{c}}$ \\
\hline $\mathbf{T}_{8}$ & $\begin{array}{l}\text { Carbofuran 3 G @ } 10 \text { kg/ha } \\
(\mathrm{SA})\end{array}$ & $\begin{array}{c}0.20 \\
(0.83)^{\mathrm{f}}\end{array}$ & $\begin{array}{c}0.27 \\
(0.87)^{\mathrm{e}}\end{array}$ & $\begin{array}{c}0.40 \\
(0.94)^{\mathrm{e}}\end{array}$ & $0.29^{\mathrm{f}}$ & $\begin{array}{c}0.20 \\
(0.84)^{\mathrm{e}}\end{array}$ & $\begin{array}{c}0.33 \\
(0.91)^{\mathrm{c}}\end{array}$ & $\begin{array}{c}0.00 \\
(0.71)^{\mathrm{e}}\end{array}$ & $0.18^{\mathrm{c}}$ \\
\hline $\mathbf{T}_{9}$ & Untreated check & $\begin{array}{c}1.27 \\
(1.33)^{\mathrm{a}}\end{array}$ & $\begin{array}{c}1.60 \\
(1.45)^{\mathrm{a}}\end{array}$ & $\begin{array}{c}1.53 \\
(1.43)^{\mathrm{a}}\end{array}$ & $1.47^{\mathrm{a}}$ & $\begin{array}{c}1.60 \\
(1.44)^{\mathrm{a}}\end{array}$ & $\begin{array}{c}1.83 \\
(1.52)^{\mathrm{a}}\end{array}$ & $\begin{array}{c}1.70 \\
(1.48)^{\mathrm{a}}\end{array}$ & $1.71^{\mathrm{a}}$ \\
\hline & & 0.04 & 0.04 & 0.04 & & 0.06 & 0.05 & 0.04 & \\
\hline & & 0.12 & 0.11 & 0.11 & & 0.18 & 0.14 & 0.11 & \\
\hline & & 6.11 & 5.11 & 5.33 & & 8.55 & 6.36 & 5.07 & \\
\hline & & $*$ & $*$ & $*$ & & $*$ & $*$ & $*$ & \\
\hline
\end{tabular}

Figures in the parentheses are $\sqrt{x}+0.5$ transformed values; DAE $=$ Days after emergence.

Means in the columns followed by the same alphabet do not differ significantly by DMRT ( $\mathrm{P}=0.05)$; SA=Soil application; $\mathrm{ST}=\mathrm{Seed}$ treatment 
Table.4 Evaluation of organics, botanicals and insecticides on the yield parameters of little millet during kharif and rabi (2015-16)

\begin{tabular}{|c|c|c|c|c|c|c|c|}
\hline \multirow{2}{*}{ Tr. No. } & \multirow{2}{*}{ Treatments } & \multicolumn{2}{|c|}{ Grain yield (q/ ha) } & \multicolumn{2}{|c|}{ Fodder yield (q/ ha) } & \multicolumn{2}{|c|}{ B: C ratio } \\
\hline & & Kharif & Rabi & Kharif & Rabi & Kharif & Rabi \\
\hline $\mathrm{T}_{1}$ & Neem cake @ 250 kg/ ha (SA) & $7.58^{\mathrm{d}}$ & $7.91^{\mathrm{d}}$ & $56.67^{\mathrm{d}}$ & $68.46^{\mathrm{bcd}}$ & 1.15 & 1.34 \\
\hline $\mathrm{T}_{2}$ & Castor cake @ 250 kg/ha (SA) & $6.85^{\mathrm{d}}$ & $7.16^{\mathrm{d}}$ & $46.75^{\mathrm{e}}$ & $62.68^{\mathrm{de}}$ & 0.72 & 0.94 \\
\hline $\mathrm{T}_{3}$ & Pongamia cake @ 250 kg/ha (SA) & $7.31^{\mathrm{d}}$ & $7.48^{\mathrm{d}}$ & $49.00^{\mathrm{e}}$ & $64.45^{\text {cde }}$ & 0.67 & 0.85 \\
\hline $\mathrm{T}_{4}$ & Rice hull ash @ 1t/ha (SA) & $9.41^{\mathrm{c}}$ & $9.02^{c}$ & $58.75^{\mathrm{cd}}$ & $68.38^{\mathrm{bcd}}$ & 1.84 & 1.96 \\
\hline $\mathrm{T}_{5}$ & NSKE@ $9 \%$ & $10.12^{\mathrm{bc}}$ & $10.46^{\mathrm{b}}$ & $62.78^{\mathrm{c}}$ & $72.65^{b}$ & 2.52 & 2.85 \\
\hline $\mathrm{T}_{6}$ & Azadirachtin@1\% & $9.98^{\mathrm{c}}$ & $9.86^{\mathrm{bc}}$ & $62.39^{\mathrm{cd}}$ & $71.28^{\mathrm{bc}}$ & 2.14 & 2.32 \\
\hline $\mathrm{T}_{7}$ & Imidacloprid 70 WS @ 5 g/ kg of seed (ST) & $12.01^{\mathrm{a}}$ & $12.07^{\mathrm{a}}$ & $78.99^{\mathrm{a}}$ & $81.54^{\mathrm{a}}$ & 3.28 & 3.43 \\
\hline $\mathrm{T}_{8}$ & Carbofuran 3 G @ 10 kg/ha (SA) & $10.95^{\mathrm{b}}$ & $11.56^{\mathrm{a}}$ & $72.16^{\mathrm{b}}$ & $82.89^{\mathrm{a}}$ & 2.83 & 3.25 \\
\hline \multirow[t]{5}{*}{$\mathrm{T}_{9}$} & Untreated check & $5.25^{\mathrm{e}}$ & $5.96^{\mathrm{e}}$ & $40.63^{f}$ & $57.55^{\mathrm{e}}$ & 1.08 & 1.66 \\
\hline & S.Em \pm & 0.29 & 0.35 & 1.99 & 2.62 & & \\
\hline & CD @ 0.05 & 0.86 & 1.04 & 5.96 & 7.85 & & \\
\hline & $\mathrm{CV} \%$ & 5.66 & 6.64 & 5.86 & 6.48 & & \\
\hline & F test & $*$ & $*$ & * & * & & \\
\hline
\end{tabular}

SA- Soil application; ST- Seed treatment; Means in the columns followed by the same alphabet do not differ significantly by DMRT ( $\mathrm{P}=0.05)$ 
Highest B: C ratio was obtained from imidacloprid seed treatment @ $5.0 \mathrm{ml} / \mathrm{kg}$ seed in both the kharif (3.28) and rabi (3.43) followed by carbofuran 3G @ $10 \mathrm{~kg} / \mathrm{ha}$ with $\mathrm{B}$ : $\mathrm{C}$ ratio of 2.83 during kharif and 3.25 during rabi (Table 4). Among the botanicals, NSKE@ $5 \%$ was the best by recording B: C ratio of 2.52 and 2.85 during kharif and rabi, respectively. In the present study application of organics was ineffective against shoot fly except neem cake @ $250 \mathrm{~kg} /$ ha which recorded 1.15 and $1.34 \mathrm{~B}$ : $\mathrm{C}$ ratio during kharif and rabi, respectively. The present findings are in consonance with those of Kumar and Channaveerswami (2015) who reported seed treatment with imidacloprid 600 FS @ $5 \mathrm{ml} / \mathrm{kg}$ of seed against little millet shoot fly recorded significantly higher seed yield per ha $(2165 \mathrm{~kg})$ and dry matter production at harvest $\left(1595 \mathrm{~g} / \mathrm{m}^{2}\right.$.

\section{Acknowledgement}

The author is thankfully acknowledged for providing financial assistance received from INSPIRE fellowship sponsored by Department of Science and Technology (DST), New Delhi.

\section{References}

Gomez, K. A., and Gomez, A. A., 1976. Statistical Procedure for Agriculltural Research $\left(2^{\text {nd }} E d\right.$.). A Willey Inter science Publication, New York. p. 680. Jagadish, P. S., Murali, P. R. and Seetharam, A., 1995. Evaluation of germplasm lines and cultural practice on the incidence of little millet shoot fly, Atherigona pulla Wiede. Environ. Edu. Res., Tokyo Gakungie Univ. No. 7-45-47.

Kumar, D. A., and Channaveerswami, A. S., 2015, Pre and post emergence control measures for shoot fly incidence and its influence on seed yield of little millet (Panicum sumatrense). J. Experimental Zool., 18(2): 811-814.

Mashwani, M. A., Ullah, F., Sattar, S., Ahmad, S. and Anwar Khan, M., 2011. Efficacy of different insecticides against maize stem borer, Chilo partellus (Swinhoe) (lepidoptera; pyralidae) at Peshawar and South Valleys of Khyber, Pakhtunkhwa, Pakistan. Sarhad J. Agric., 27(3): 459-465.

Parteti, S. R., Kalinkar, A. S., Gurve, S. S. and Gawande, R. W., 2014. Efficacy of botanicals for the management of sorghum shoot fly. Trends in Biosci, 7(17): 2566-2569.

Rajesh, K., Singh, M. and Narendra, K., 2016. Estimation of losses due to shoot fly, Atherigona soccata (Rondani) in spring maize. Indian J. Entomol., 78(1): 28-31.

Tandi, B. L., and Bajiya, R. S., 2013. Management of shoot fly and stem borer in pearl millet. Indian J. Appl. Entomol., 27(1): 73-75.

\section{How to cite this article:}

Ravulapenta Sathish, M. Manjunatha and Rajashekarappa, K. 2017. Effect of Organic Amendments, Botanicals and Insecticides against Little Millet Shoot Fly, Atherigona pulla (Wiedemann). Int.J.Curr.Microbiol.App.Sci. 6(10): 2196-2203. doi: https://doi.org/10.20546/ijcmas.2017.610.260 